\title{
SERIOUS GAMES: ESCENARIOS LÚDICOS PARA EL DESARROLLO DE LAS INTELIGENCIAS MÚLTIPLES EN ESCOLARES DE PRIMARIA
} \author{
MULTIPLE INTELLIGENCES IN PRIMARY SCHOOL CHILDREN \\ María Esther Del-Moral-Pérez; emoral@uniovi.es \\ Universidad de Oviedo \\ Alba Patricia Guzmán-Duque; albapatrig@gmail.com \\ Unidades Tecnológicas de Santander (Colombia) \\ Laura Carlota Fernández; karlotamail@gmail.com \\ Universidad de Oviedo
}

SERIOUS GAMES: LEARNING THROUGH PLAY FOR THE DEVELOPMENT OF

\section{RESUMEN}

La presente investigación constata cómo determinados serious games pueden contribuir al desarrollo de las Inteligencias Múltiples en escolares de primaria. Se describe una experiencia innovadora apoyada en el uso sistemático semanal de un videojuego con alumnos de primaria $(\mathrm{N}=101)$. Los resultados cotejados con un grupo de control muestran incrementos significativos en sus inteligencias -utilizando el instrumento (Prieto y Ferrándiz, 2001) antes y después de la experiencia- especialmente la matemática y la viso-espacial en las niñas, y la lingüística y la interpersonal en los niños, siendo ellas las más beneficiadas globalmente. Ello invita a reflexionar sobre la necesaria cualificación docente para aprovechar las oportunidades de estos instrumentos lúdicos, reduciendo el esfuerzo cognitivo que suponen algunos aprendizajes, y lograr rendimientos exitosos.

Palabras clave: Videojuegos, inteligencias múltiples, educación primaria, competencias sociales, aprendizaje interactivo.

\begin{abstract}
This research shows how certain serious games can contribute to the development of multiple intelligences at primary school. We describe an innovative experience supported by the routine weekly use of a video game with elementary school students $(\mathrm{N}=101)$. Results collated with a control group showed significant increases in their minds -using the instrument (Prieto \& Ferrándiz, 2001) before and after the experience- especially mathematics and viso-space in girls, and linguistics and interpersonal in children, where girls benefit most globally. It invites to reflect on the required teaching qualification for these instruments ludic, this tools are reducing the cognitive effort involving some learning opportunities, and achieve successful yields.
\end{abstract}

Keywords: Videogames, multiple intelligences, primary education, social skills, interactive learning. 


\section{INTRODUCCIÓN}

La Teoría de las Inteligencias Múltiples (IM) (Gardner, 2005) apuesta por un modelo de enseñanza centrado en el aprendiz, orientado al desarrollo de habilidades que impliquen globalmente a todas las inteligencias: lingüística, musical, lógico-matemática, viso-espacial, corporal-cinestésica, interpersonal, intrapersonal y naturalista. Considerándolas susceptibles de mejora cualitativa dentro de un entorno cultural rico en estímulos, a partir de estrategias y actividades sistemáticas.

Amstrong (1994), Prieto y Ballester (2003), y, Prieto y Ferrándiz (2001), explican que las IM pueden potenciarse mediante actividades específicas. Recientes investigaciones demuestran el incremento de las IM a partir de distintas prácticas de enseñanza (Saricaoglu \& Arikan, 2009), algunas apoyadas en entornos virtuales (Riha \& Robles-Piña, 2009), otras en juegos digitales (Sanford \& Madill, 2007; Schaaf, 2012).

De modo similar, extrapolando sus propuestas al contexto de los serious games, la presente investigación se propuso constatar si éstos podían contribuir a desarrollar las IM en escolares de primaria, aprovechando su motor de juego y la motivación extrínseca que suponen sus incentivos multisensoriales (recreación y banda sonora, melodías, resolución gráfica, colorido, locuciones,...).

Hoy, la escuela es consciente del contexto tecnológico en que viven los menores y es permeable a las oportunidades que algunos videojuegos ofrecen para el aprendizaje: adquisición de destrezas y habilidades, promoción de la salud, socialización de la infancia y adolescencia... (Pindado, 2005), así como para ayudar a comprender el funcionamiento socio-organizativo de la sociedad, a escudriñar el papel que juegan las convenciones sociales, etc. Además, son numerosas las voces que reclaman nuevas formas de alfabetización (Gómez del Castillo, 2007; Marín \& García, 2005), de intercambio comunicativo y de pensamiento para explotar estos novedosos instrumentos digitales como potentes recursos didácticos en el aula.

\section{SERIOUS GAMES Y DESARROLLO DE INTELIGENCIAS MÚLTIPLES}

Existen interesantes experiencias de integración de videojuegos en contextos formativos (Cortés, García \& Lacasa, 2012; Del Castillo, Herrero, García, Checa \& Monjelat, 2012; Sung \& Hwang, 2013) con diversos fines: refuerzo de aprendizajes, alfabetización digital, simulación de procesos sociales, fomento de la experimentación y curiosidad, desarrollo de capacidades (resolución de problemas, construcción de historias, toma de decisiones, etc.), siempre vinculados a la naturaleza del videojuego, sus temáticas, las actividades que incluye y los márgenes de libertad que permite a los jugadores mejorar su aprendizaje (Robertson, 2013; Vos, Van der Meijden \& Denessen, 2011). 
La inteligencia lingüística, o capacidad para comunicarse mediante lenguaje oral o escrito, puede activarse con videojuegos que impliquen conversaciones y prácticas lingüísticas. Thorne, Black y Sykes (2009) constatan el incremento de competencias lingüísticas de los jugadores que interaccionan con videojuegos online.

Por su parte, la inteligencia lógico-matemática, o capacidad de razonamiento deductivo (utilización de números, cantidades, distancias, relaciones entre objetos, etc.), puede potenciarse con el apoyo de videojuegos orientados al cálculo mental (game-training), logrando mejorarla (Chang, Wu, Weng y Sung, 2012). Hay experiencias exitosas con adultos (Basak, Boot, Voss \& Kramer, 2008) y sujetos con necesidades educativas especiales (Papastergiou, 2009).

La inteligencia corporal-cinestésica, o capacidad para utilizar el cuerpo para expresar ideas y sentimientos, producir o transformar cosas, provocar desplazamientos, saltos, coreografías de bailes, etc., es favorecida con el exergaming, especialmente con la wii, simulando deportes (tenis, esquí...). Algunos pueden generar una actividad equiparable al paseo y mejorar las destrezas motoras específicas del deporte que se ejercite (Graf, Pratt, Hester \& Short, 2009).

En mayor medida, la inteligencia viso-espacial, o habilidad para percibir y reconocer el entorno espacial donde se desenvuelve el sujeto, capacidad para transformar, interpretar y plasmar las percepciones recibidas: reconocimiento de mapas, ubicación en entornos 3D, identificación y combinación de códigos visuales..., es susceptible de desarrollo implícito con videojuegos de alta calidad gráfica con recreaciones bi o tridimensionales, imágenes, vídeos, secuencias fílmicas que presentan espacios cotidianos; $y$, explícitamente, con aplicaciones que inviten a la realización de diseños, dibujos, construcción de puzzles y rompecabezas... Llorca (2009), Greenfield (2009), y, Yang y Chen (2010) afirman que algunos videojuegos favorecen las habilidades espaciales, y Korallo, Foreman, Boyd-Davis, Moar y Coulson (2012) describen los efectos positivos a nivel cognitivo vinculados a la memoria.

La inteligencia naturalista, o habilidad para discriminar y clasificar los organismos vivos, identificar ecosistemas, comprender el mundo natural tras observarlo..., se puede incrementar con videojuegos que permiten la experimentación, como Norrath (Castronova, 2006) o Spore, orientado a crear nuevas especies, logrando resultados óptimos (García, Cortés \& Martínez, 2011).

La inteligencia musical, o capacidad para percibir, discriminar, transformar y expresar formas musicales, es posible potenciarla con videojuegos que integren simuladores para la creación de composiciones musicales a partir de diferentes instrumentos y matices culturales; 0 , con otros estilos karaoke, que permiten crear o poner letra a melodías de canciones. O, a partir de aquellos que posibilitan la composición musical, creación de coreografías, etc. Los videojuegos con bandas sonoras propias, tocadas por prestigiosas orquestas sinfónicas, ayudan a educar el gusto por la música, reconocer obras clásicas, etc., constituyéndose en propulsores de la educación estética.

La inteligencia interpersonal, o capacidad de percibir y establecer distinciones en los estados de ánimo, las intenciones, las motivaciones, y los sentimientos de otras personas, puede activarse 
con videojuegos de simulación social. Greitmeyer y Osswald (2010) demostraron que aplicaciones educativas apoyadas en videojuegos de componente prosocial pueden lograr conductas prosociales en jugadores de riesgo.

Para incrementar la inteligencia intrapersonal, o conocimiento propio de las fortalezas y debilidades que cada sujeto tiene de sí mismo junto a su habilidad para actuar y tomar decisiones consecuentes al respecto, desempeñado roles y funciones acordes a sus destrezas..., existen múltiples videojuegos que contribuyen a la superación personal y al desarrollo profesional, como juegos de rol y simuladores sociales para el entrenamiento en la toma de decisiones, habilidades para la negociación, mediación en conflictos, etc. Botero (2011) enfatiza el poder de determinados videojuegos para el entrenamiento de habilidades directivas $y$ liderazgo en empresas.

Los contextos formales educativos, a menudo, olvidan potenciar las habilidades emocionales y sociales, que pueden contribuir a predecir el éxito social y personal futuro, como afirman Ferrándiz, Hernández, Bermejo, Ferrando y Sáinz (2012), por ello, los videojuegos bien pueden considerarse unos instrumentos adecuados para ello.

Finalmente, si bien existen investigaciones que intentan medir el efecto de determinados videojuegos en el incremento de destrezas y habilidades de los sujetos, se precisa de mayor rigor para analizar su influencia en el desarrollo de las ocho inteligencias, pues no todos son apropiados, sólo aquellos que contemplan actividades sistemáticas y contribuyen de forma continua a su entrenamiento. De ahí, que la selección de videojuegos, en función de los contenidos y las actividades cognitivas que proponen, sea fundamental para garantizar el desarrollo de las dimensiones específicas de cada una de las inteligencias.

\section{INTEGRACIÓN DEL VIDEOJUEGO NARABA EN EL CONTEXTO ESCOLAR}

Echeverría et al. (2011) afirman que la integración de actividades colaborativas apoyadas en juegos implica una rigurosa selección para promover aprendizajes de forma explícita. Las herramientas para evaluar la viabilidad de la integración curricular y la explotación didáctica de videojuegos, ofrecidas por Del Moral y Villalustre (2012), facilitaron la selección del videojuego para la finalidad de la investigación. Así pues, evaluada la potencialidad educativa de distintos videojuegos o serious games, sus actividades lúdicas, recursos expresivos, fórmulas de interacción..., y determinadas las inteligencias que preferentemente podían desarrollar, se seleccionó el videojuego Naraba (Micronet, 2010) por considerar que cumplía con unos prerrequisitos básicos: abordar contenidos curriculares propios de las distintas áreas de educación primaria sin perder su potencial lúdico, explotando su jugabilidad a partir de recursos técnicos y narrativos que le dotan de gran atractivo para los alumnos.

Naraba propone la realización de actividades para superar retos que exigen un modo de interacción concreto, y ofrece la oportunidad de ejercitar explícitamente diferentes inteligencias. Incluye actividades específicas directamente relacionadas con varias materias del 
currículum de Educación Primaria (LOE, 2006), se tratan temas como la salud y respeto al medio ambiente. Se orienta a favorecer el desarrollo de distintas habilidades cognitivas y a la adquisición de destrezas básicas, fundamentales para sentar las bases de nuevos aprendizajes. Consta de cuarenta misiones o actividades que el jugador debe realizar, agrupadas en torno a tres áreas: conocimiento del medio (19), matemáticas (12) y educación artística (9). Todas apelan a estrategias didácticas que potencian en mayor o menor medida las distintas inteligencias (Gardner, 2005). La Figura 1 muestra el peso concedido al desarrollo de cada inteligencia por el conjunto de las misiones que incluye el videojuego seleccionado.

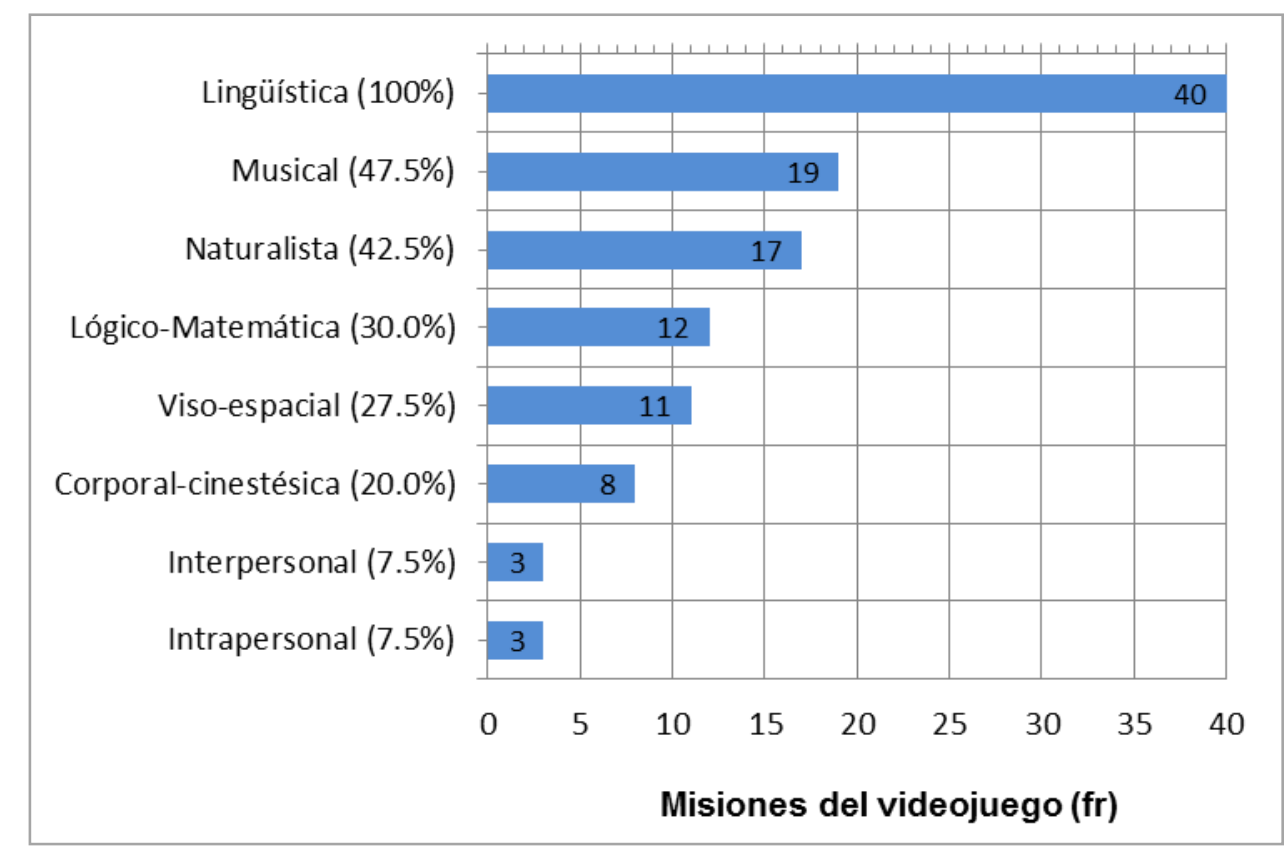

Figura 1. Distribución porcentual de las inteligencias potenciadas con las misiones del juego.

Al evaluar el videojuego se observó que las cuarenta misiones implicaban a varias inteligencias simultáneamente. La Figura 2 muestra la interfaz del juego y ejemplos de algunas misiones. 


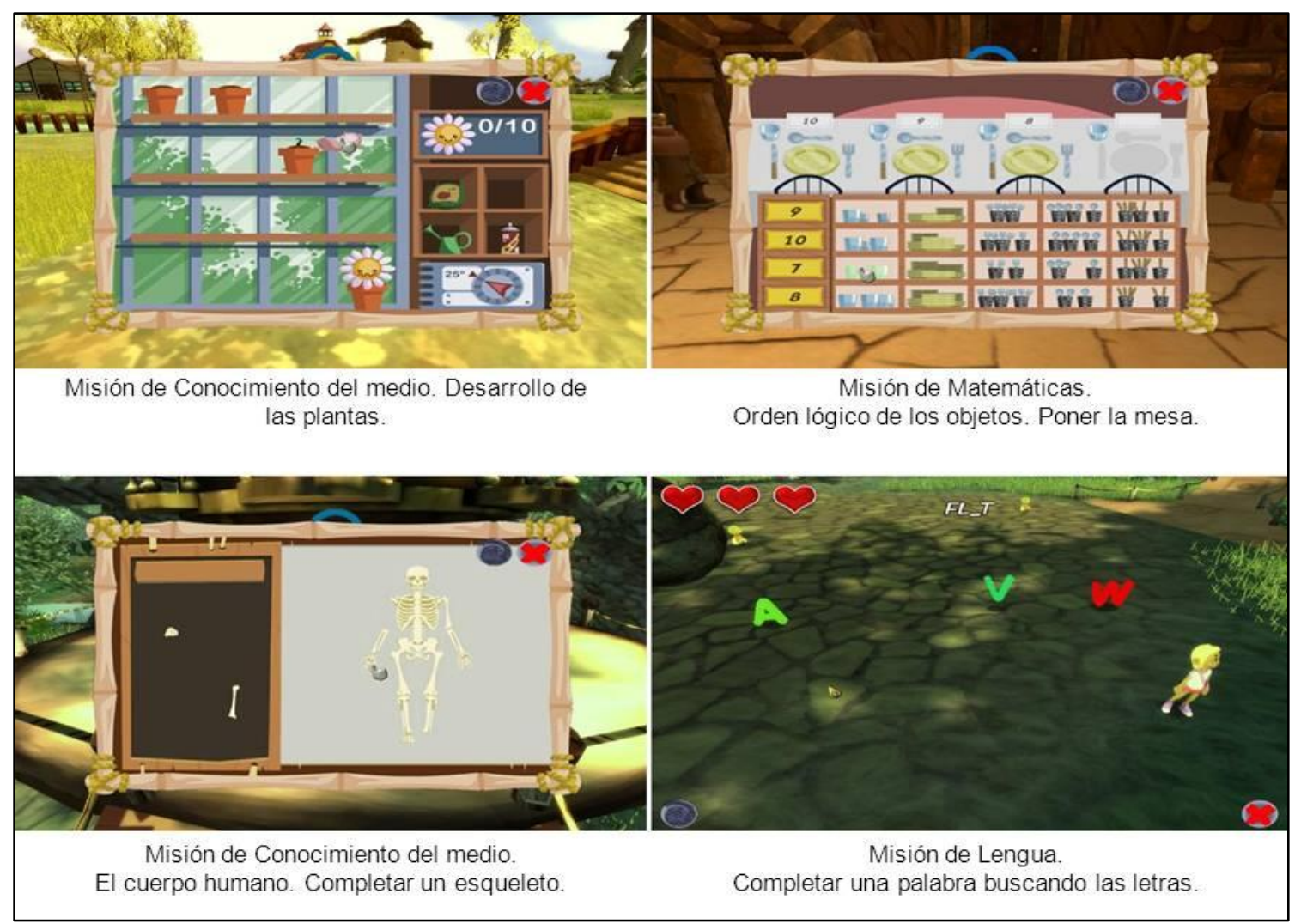

Figura 2. Ejemplos de misiones del videojuego Naraba.

Todas las misiones contribuyen a desarrollar la inteligencia lingüística al demandar el uso de la lengua oral y escrita para sonorizar y subtitular los diálogos de los personajes, ejercitando las habilidades comunicativas. Diecisiete potencian la naturalista al favorecer la observación, investigación, formulación, comprobación de hipótesis, explicación de resultados, y transversalmente, abordar temas de salud y desarrollo personal, seres vivos, conservación del medio ambiente, materia, energía, personas, culturas y organización social. Once misiones desarrollan la inteligencia viso-espacial al incidir en relaciones y representaciones espaciales (percepción visual), producción y apreciación artística. Otras apelan a la intrapersonal: autoconcepto, autocontrol y autodisciplina, interacción con otros, solución de conflictos, asunción de distintos roles sociales y liderazgo. Ocho activan la corporal-cinestésica, concretamente la motricidad fina, al usar el teclado y el ratón para accionar los elementos interactivos. Doce misiones fomentan la lógico-matemática (reconocimiento de tamaños, colores, formas, comparaciones, clasificaciones, cantidades y razonamiento analógico), trabajan números y operaciones, medidas, estimación y cálculo de magnitudes, geometría, tratamiento de la información, azar y probabilidad. Y diecinueve favorecen la inteligencia musical, entrenan la percepción, producción, composición e interpretación musical, y memorización de recitados rítmicos.

Este exhaustivo estudio del videojuego Naraba-World permitió visibilizar la concordancia entre las misiones propuestas y las competencias de Primaria que desarrollan, vinculadas a la lengua 
(castellano e inglés), matemáticas, conocimiento del medio y educación artística, junto a las inteligencias que potencian explícita o implícitamente. Factores determinantes para considerarlo idóneo para la investigación.

El proceso didáctico llevado a cabo para insertar el videojuego en las aulas de primaria requirió de una planificación previa para hacer converger los contenidos de las distintas áreas (Lengua, Conocimiento del Medio, Matemáticas y Educación Artística) con las clases prácticas dedicadas a la realización de las misiones o actividades propuestas en el videojuego seleccionado. Lográndose establecer una correspondencia entre los contenidos curriculares y las actividades lúdicas integradas en el Naraba. A pesar de que se siguió el orden de la programación del curso, algunas misiones del juego obligaban a las docentes a adelantar contenidos, para ajustarlos a las demandas impuestas por el videojuego, y con ello permitir a los alumnos avanzar en el mismo.

Las sesiones dedicadas al videojuego se concentraban al final de la semana, los alumnos jugaban una hora semanal los viernes. Adquirían nuevos aprendizajes de forma lúdica y de buen grado, motivados por la urgencia de completar las misiones del juego. Y en el caso de que los contenidos ya se hubieran impartido en clase, el juego servía de repaso para su fijación. En definitiva, el uso del videojuego permitió la adquisición de nuevos conocimientos vinculados a las misiones del juego, además de reforzar aquellos ya aprendidos.

El proceso didáctico llevado a cabo para insertar el videojuego en las aulas de primaria requirió de una planificación previa para hacer converger los contenidos de las distintas áreas (Lengua, Conocimiento del Medio, Matemáticas y Educación Artística) con las clases prácticas dedicadas a la realización de las misiones propuestas en el videojuego seleccionado. Lográndose establecer una correspondencia entre los contenidos curriculares y las actividades lúdicas integradas en el Naraba. A pesar de que se siguió el orden de la programación del curso, algunas misiones del juego obligaban a las docentes a adelantar contenidos, para ajustarlos a las demandas impuestas por el videojuego, y con ello permitir a los alumnos avanzar en el mismo.

Las sesiones dedicadas al videojuego se concentraron al final de la semana, los alumnos jugaban una hora semanal los viernes. Adquirían nuevos aprendizajes de forma lúdica y de buen grado, motivados por la urgencia de completar las misiones del juego. Y en el caso de que los contenidos ya se hubieran impartido en clase, el juego servía de repaso para su fijación. En definitiva, el uso del videojuego permitió la adquisición de nuevos conocimientos vinculados a las misiones del juego, además de reforzar aquellos ya aprendidos.

\section{METOdOLOGÍA}

Para constatar si la utilización planificada y sistemática del serious game Naraba podía contribuir al desarrollo de las Inteligencias Múltiples en escolares de primaria, se analizó la experiencia desarrollada durante el curso 2012-13, concretada en la dedicación de una hora semanal al juego, Ilevada a cabo en la Escuela de Primaria Gesta I de Oviedo (Asturias-España). 


\subsection{Participantes}

La muestra estuvo integrada por 101 alumnos, de la cual se extrajeron 20 sujetos para el grupo de control $(G C), 81$ formaron parte del grupo experimental (GE), distribuidos como indica la Tabla 1, a quienes se midió su nivel de IM al inicio de la experiencia (test) y al final de la misma (retest), con el fin de comprobar a posteriori la evolución operada en las inteligencias de los sujetos tras participar en la experiencia con el juego.

\begin{tabular}{lccccc}
\hline \multirow{2}{*}{ Curso/Sexo } & \multicolumn{2}{c}{ Niños } & \multicolumn{2}{c}{ Niñas } & \multirow{2}{*}{ Total } \\
\cline { 2 - 5 } & Participantes & G.C. & Participantes & G.C. & \\
\cline { 3 - 6 } Primero de Primaria & $20(48.8 \%)$ & $5(50.0 \%)$ & $17(42.5 \%)$ & $5(50.0 \%)$ & $47(46.5 \%)$ \\
Segundo de Primaria & $21(51.2 \%)$ & $5(50.0 \%)$ & $23(57.5 \%)$ & $5(50.0 \%)$ & $54(53.4 \%)$ \\
Total & $41(50.6 \%)$ & $10(50.0 \%)$ & $40(49.4 \%)$ & $10(50.0 \%)$ & $101(100.0 \%)$ \\
\hline
\end{tabular}

Tabla 1. Distribución de la muestra $(N=101)$ atendiendo a las variables curso y sexo.

\subsection{Instrumento de recogida de información}

Se eligió el Cuestionario de Evaluación de Inteligencias Múltiples de Prieto y Ferrándiz (2001) pues ya fue validado y facilitaba las tareas de los docentes para el seguimiento individualizado de la evolución de cada alumno. El cual está integrado por ochenta ítems o indicadores cualitativos -diez por inteligencia- , ayudó a determinar el nivel que los sujetos presentaban en cada una, utilizando una escala del 1 al 4 (1=muy bajo; 2=bajo; 3=alto; 4=muy alto), que permitió realizar observaciones sobre los progresos individuales relativos a las competencias y/o habilidades que sirvieron para ponderar cada inteligencia, así:

- Lingüística, evaluada a partir de competencias comunicativas referidas al lenguaje oral y escrito, las habilidades para la lectura, ortografía y vocabulario de cada sujeto.

- Naturalista, mide su nivel de competencia en relación al pensamiento científico experimentación e indagación- y al estudio de las ciencias.

- Viso-espacial, se examina mediante ítems que permiten identificar las habilidades artísticas -representación y exploración de formas, colores...-, y viso-espaciales que poseen.

- Corporal-cinestésica, medida a partir de las habilidades físicas y deportivas, del desarrollo de la motricidad gruesa y fina, y del lenguaje corporal que manifiestan.

- Musical, evaluada a través de las actitudes hacia la música, la capacidad para cantar, reconocer sonidos, identificar estilos musicales, expresión rítmica y musical, etc. 
- Lógico-matemática, medida a partir de las competencias para resolver problemas, cálculo, aritmética, categorización de objetos, pensamiento abstracto y procesos cognitivos de orden superior que se activan.

- Interpersonal, evaluada a través de la capacidad de liderazgo, habilidades para trabajar en equipo, mediación y empatía con otros.

- Intrapersonal, inferida a partir del grado de autoconocimiento que poseen, facilidad para trabajar autónomamente y de otras competencias manifestadas (constancia, rigor...).

\subsection{Procedimiento}

El estudio se centró en constatar si la experiencia de integración didáctica, sistemática y planificada, de un videojuego en aulas de primaria, podría favorecer el incremento de las IM del alumnado. A priori se entendía que la clave, entre otros aspectos, radicaría en las oportunidades que el videojuego seleccionado ofrecía para el aprendizaje. Ello obligó a optar por un videojuego que permitiese alcanzar estas expectativas. Así, tras evaluar varios y clasificar sus actividades en relación a las inteligencias que podían ejercitar, se acordó que Naraba podría ajustarse al objetivo. Posteriormente, se presentó a los docentes, quienes valoraron su pertinencia para implementarlo en sus aulas, dedicando una hora semanal al videojuego durante el curso lectivo 2012-13.

Por un lado, se adoptó una metodología cualitativa, en tanto estudio de caso implementado en una escuela ( $\mathrm{N}=101)$, cuyo seguimiento fue realizado por las docentes de primaria en calidad de investigadoras participantes -con el asesoramiento experto externo- quienes registraron el nivel de cada alumno, en las ocho inteligencias tanto al inicio de la experiencia (Fase I Test: octubre 2012) como al concluirla (Fase II Retest: mayo 2013), utilizando el mencionado cuestionario.

El test de Prieto y Ferrándiz (2001) presenta un total de 80 ítems con un marcado carácter cualitativo -diez por cada inteligencia-, a partir de los cuales, las docentes debían valorar las habilidades y/o competencias específicas de cada alumno respecto a los demás que conforman el grupo, mediante una escala tipo likert, tanto al inicio de la experiencia como al concluir la misma. De modo que se obtuvieron dos medidas diferentes, pero siempre relativas a la percepción subjetiva de las docentes respecto a la situación individual de cada sujeto en las ocho inteligencias y en comparación con el resto de sujetos del grupo, antes y después de la experiencia llevada a cabo con el videojuego. Lógicamente, fueron las tutoras las que realizaron esta ponderación cualitativa, al ser ellas las que mejor conocían la evolución global de la totalidad del alumnado implicado en la experiencia.

El test de Prieto y Ferrándiz (2001) presenta un total de 80 ítems con un marcado carácter cualitativo -diez por cada inteligencia-, a partir de los cuales, las docentes debían valorar las habilidades y/o competencias específicas de cada alumno respecto a los demás que conforman el grupo, mediante una escala tipo likert, tanto al inicio de la experiencia como al concluir la misma. De modo que se obtuvieron dos medidas diferentes, pero siempre relativas a la percepción subjetiva de las docentes respecto a la situación individual de cada sujeto en las ocho inteligencias y en comparación con el resto de sujetos del grupo, antes y después de la experiencia llevada a cabo con el videojuego. Lógicamente, fueron las tutoras las que realizaron 
esta ponderación cualitativa, al ser ellas las que mejor conocían la evolución global de la totalidad del alumnado implicado en la experiencia.

Posteriormente, dichos datos se volcaron en una base de datos para su posterior análisis estadístico para constatar la influencia real del videojuego en el incremento de las inteligencias del alumnado, utilizando técnicas estadísticas descriptivas para explicar su variación en las dos fases. La Figura 3 muestra el proceso de la investigación y los hitos más significativos que la vertebraron:

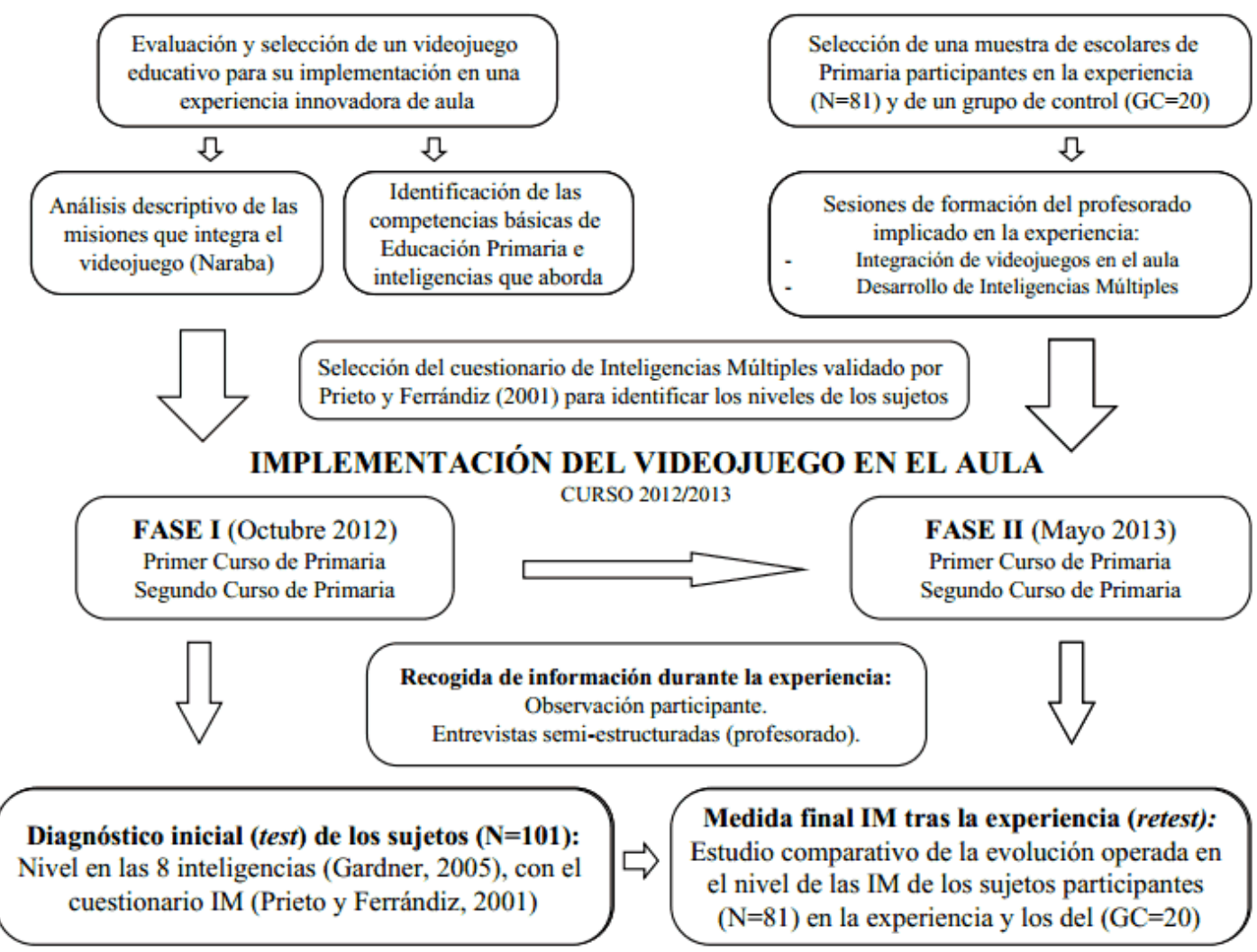

Figura 3. Proceso de la investigación y fases que la componen.

En la Fase I se identificó el nivel de todos los sujetos $(\mathrm{N}=101)$ en cada inteligencia antes de iniciar la experiencia con el videojuego. Siete meses después, concluida ésta, en la Fase // se volvió a medir el nivel de cada sujeto -participantes (GE) y GC-, con objeto de estimar comparativamente su evolución e identificar el posible efecto del videojuego. El tratamiento estadístico (prueba $t$-student) de ambas medidas (test-retest), constató la existencia de diferencias significativas. Un análisis más pormenorizado permitió comparar las medias en función de las variables "curso" y "sexo" en cada Fase, para determinar si podían ser las responsables del cambio. El mismo tratamiento se realizó con el GC para establecer en qué 
medida la participación en la experiencia podría contribuir a explicar el incremento de las inteligencias. Los estadísticos se estimaron con el 95.0\% de confiabilidad. Se empleó el paquete estadístico SPSS (v.18).

\section{ANÁLISIS DE RESULTADOS Y DISCUSIÓN}

\subsection{Competencias de las inteligencias}

Las valoraciones de los sujetos ( $N=101)-51$ niños (50.50\%) y 50 niñas (49.50\%)- de entre 7 y 8 años, se hicieron para las ocho inteligencias según el cuestionario de Prieto y Ferrándiz (2001) a partir de 80 ítems medidos con una escala de 1 a 4 (1=muy bajo; 2=bajo; 3=alto; 4=muy alto).

La Tabla 2 muestra la distribución de los sujetos según el nivel alcanzado en cada inteligencia en la Fase I. Mayoritariamente los sujetos presentan niveles muy bajos y bajos respecto a las inteligencias naturalista, matemática e interpersonal. En el nivel alto destacan en la visoespacial (54.20\%), musical (52.20\%) e intrapersonal (52.10\%). Son pocos los sujetos que presentan niveles muy altos en las inteligencias interpersonal y lingüística (9.90\%) y (9.30\%) respectivamente.

\begin{tabular}{|c|c|c|c|c|c|}
\hline Inteligencia & Fase & \% Muy bajo & \% Bajo & $\%$ Alto & $\%$ Muy alto \\
\hline \multirow{2}{*}{ Lingüística } & Fase I & 6.70 & 35.70 & 48.40 & 9.30 \\
\hline & Fase II & 5.20 & 28.60 & 51.10 & 16.70 \\
\hline \multirow{2}{*}{ Naturalista } & Fase I & 12.10 & 34.60 & 45.40 & 7.90 \\
\hline & Fase II & 8.20 & 22.10 & 52.80 & 17.70 \\
\hline \multirow{2}{*}{ Matemática } & Fase I & 10.80 & 37.40 & 46.30 & 4.80 \\
\hline & Fase II & 6.40 & 27.80 & 48.20 & 17.70 \\
\hline \multirow{2}{*}{ Visoespacial } & Fase I & 7.40 & 33.00 & 54.20 & 6.20 \\
\hline & Fase II & 4.00 & 23.60 & 51.10 & 21.70 \\
\hline \multirow{2}{*}{ Musical } & Fase I & 7.10 & 33.80 & 52.20 & 7.40 \\
\hline & Fase II & 3.40 & 25.90 & 55.10 & 15.30 \\
\hline \multirow{2}{*}{ Corporal } & Fase I & 8.00 & 37.80 & 47.00 & 7.00 \\
\hline & Fase II & 5.10 & 27.20 & 49.40 & 18.40 \\
\hline \multirow{2}{*}{ Interpersonal } & Fase I & 10.70 & 28.60 & 50.70 & 9.90 \\
\hline & Fase II & 6.70 & 20.10 & 54.30 & 19.60 \\
\hline \multirow{2}{*}{ Intrapersonal } & Fase I & 5.90 & 35.50 & 52.10 & 6.40 \\
\hline & Fase II & 3.70 & 27.40 & 56.30 & 12.20 \\
\hline
\end{tabular}

Tabla 2. Distribución porcentual de los sujetos según los niveles alcanzados en ambas Fases del Experimento. 
Concluida la experiencia (Fase II), se detecta que las nuevas valoraciones de las inteligencias de los sujetos del GE (Tabla 2) muestran un incremento generalizado respecto a la Fase I, balanceándose a los niveles de alto y muy alto. Se observa una disminución de los sujetos en los niveles muy bajo y bajo en la inteligencia naturalista. Los sujetos de los niveles más altos se concentran en la inteligencia intrapersonal (56.30\%), musical (55.10\%), interpersonal (54.30\%), naturalista (52.80\%), lingüística (51.10\%), visoespacial $(51.10 \%)$, corporal $(49.40 \%)$ y matemática (48.20\%). Finalmente, las inteligencias que susceptiblemente se incrementan más en el nivel muy alto son la visoespacial (21.70\%) seguida de la interpersonal (19.60\%).

El análisis descriptivo evidencia que algunos de los ítems que componen cada inteligencia mejoran, tras el uso del videojuego en el aula, así:

En la Lingüística se observa un incremento promedio en los ítems relacionados con la escritura (Media=2.74), las habilidades causa-efecto (Media=2.69), disfrute con las narraciones del videojuego (Media=3.11) y simulaciones (Media=3.01).

En la Naturalista destacan los sujetos en los ítems asociados a disfrutar del videojuego (Media=3.20), curiosidad (Media=2.93), comparación y clasificación (Media=2.77), predecir simulaciones (Media=2.75) y gusto por la novedad del juego (Media=3.02).

En la Fase I/ en la Matemática los sujetos registran una mejoría significativa en los ítems relacionados con cálculos mentales (Media=2.83), misiones matemáticas (Media=2.98), gusto por el juego (Media=2.95), rompecabezas (Media=2.94) y secuencias lógicas (Media=2.75).

Los sujetos en la Visoespacial (retest) ven incrementadas sus puntuaciones en los ítems que se refieren a las imágenes mentales y en la pantalla, cambiar mentalmente, animaciones, puzzles y laberintos.

En la Musical, mejoran en los ítems relacionados con recordar melodías (Media=2.86), habilidad para componer (Media=2.80) y gusto por la música (Media=2.99).

Los incrementos en la Corporal-cinestésica se evidencian en los ítems relacionados con la manipulación (Media=2.78), correr y movimientos (Media=2.80), habilidad y precisión motriz (Media=2.77), expresar con gestos (Media=2.67) y sensaciones físicas (Media=2.77).

En la Interpersonal mejoran los relacionados con su habilidad para aconsejar (Media=2.80), manejo en diversos ámbitos (Media=2.80), ayudar a los compañeros (Media=2.81), enseñar informalmente (Media=2.77), actitud mediadora (Media=2.40), capacidad de adaptación (Media=2.64) y empatía (Media=2.70).

Finalmente, en la Intrapersonal se observan incrementos significativos en los ítems relacionados con la autonomía (Media=2.77), expresión de sentimientos (Media=2.67), aprender de sus éxitos/fracasos (Media=2.68) y la autoestima (Media=2.90). 


\subsection{Comparación entre las dos fases}

Tras el contraste de los datos obtenidos en ambas fases, la Tabla 3 muestra que las inteligencias con mayor incremento, en la totalidad de los sujetos, son la Viso-espacial (Media=2.91, $\mathrm{SD}=0.59$ ); Matemática (Media=2.74, SD=0.66); Lingüística (Media=2.86, SD=0.55); corporal (Media=2.81, SD=0.79); e Interpersonal (Media=2.86, SD=0.79).

\begin{tabular}{|c|c|c|c|c|c|}
\hline \multirow{2}{*}{ Inteligencia } & \multirow{2}{*}{ Grupo } & \multicolumn{2}{|c|}{ Fase I } & \multicolumn{2}{|c|}{ Fase II } \\
\hline & & Media & SD & Media & SD \\
\hline \multirow{2}{*}{ Lingüística } & GE & 2.60 & 0.56 & 2.86 & 0.55 \\
\hline & GC & 2.90 & 1.10 & 2.60 & 1.10 \\
\hline \multirow{2}{*}{ Naturalista } & GE & 2.49 & 0.65 & 2.8 & 0.66 \\
\hline & GC & 2.90 & 1.10 & 2.50 & 1.00 \\
\hline \multirow{2}{*}{ Matemática } & GE & 2.46 & 0.57 & 2.74 & 0.66 \\
\hline & GC & 2.80 & 1.10 & 2.60 & 1.00 \\
\hline \multirow{2}{*}{ Visoespacial } & GE & 2.59 & 0.5 & 2.91 & 0.59 \\
\hline & GC & 2.80 & 1.00 & 2.50 & 0.90 \\
\hline \multirow{2}{*}{ Musical } & GE & 2.60 & 0.52 & 2.82 & 0.53 \\
\hline & GC & 2.60 & 1.00 & 2.70 & 0.80 \\
\hline \multirow{2}{*}{ Corporal } & GE & 2.53 & 0.74 & 2.81 & 0.79 \\
\hline & GC & 2.80 & 1.10 & 2.60 & 0.90 \\
\hline \multirow{2}{*}{ Interpersonal } & GE & 2.60 & 0.81 & 2.86 & 0.79 \\
\hline & GC & 2.70 & 1.00 & 2.60 & 0.90 \\
\hline \multirow{2}{*}{ Intrapersonal } & GE & 2.59 & 0.7 & 2.77 & 0.71 \\
\hline & $\mathrm{GC}$ & 2.80 & 1.10 & 2.50 & 1.00 \\
\hline
\end{tabular}

Tabla 3. Medias y desviaciones estándar de las IM obtenidas por los sujetos en ambas fases del Experimento.

En la Gráfica 1 se percibe un desplazamiento de las líneas que indica que las niñas son las más beneficiadas, en especial en las inteligencias matemática y lingüística. En cuanto a los niños, se detecta el desplazamiento en las inteligencias viso-espacial y lingüística. 


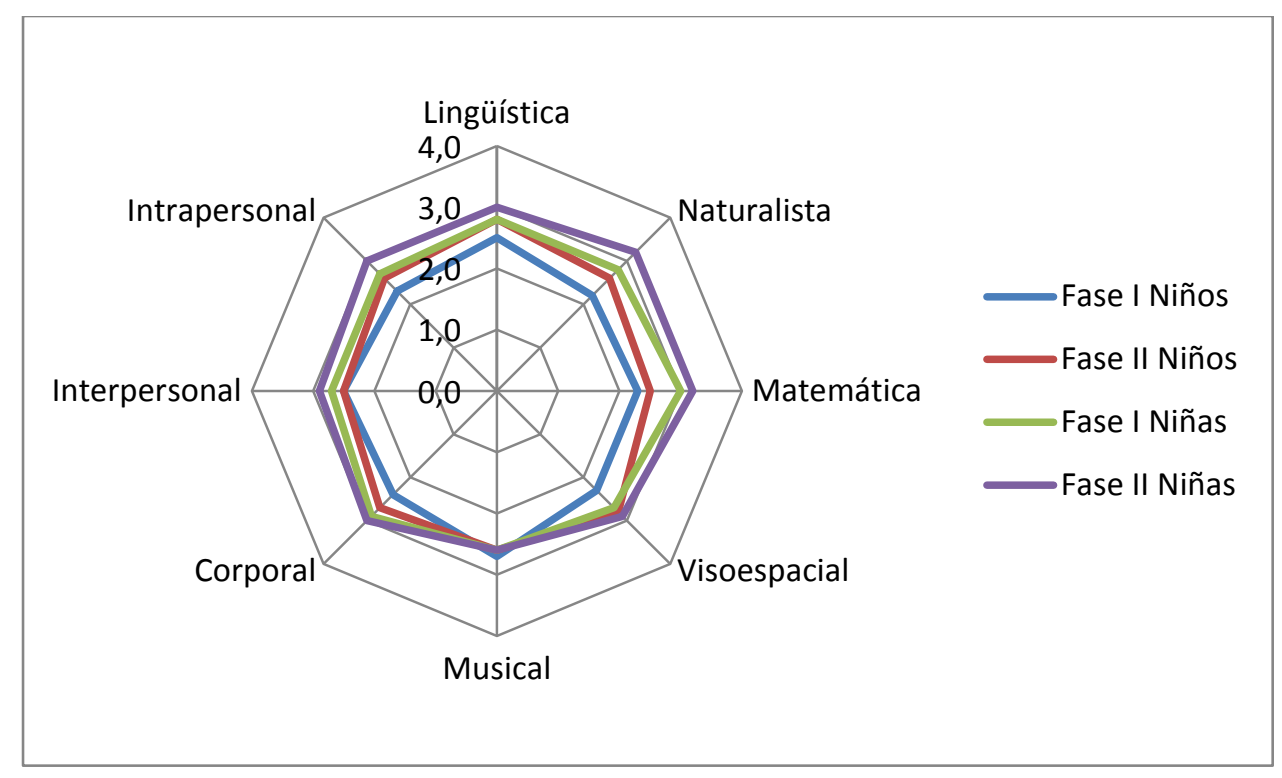

Gráfico 1. Valores medios de las Inteligencias en ambas fases, según sexo.

Con la prueba $t$-student se detecta que no existen diferencias significativas entre ambos grupos -GE y GC- (NS=95.00\%). Esto permite inferir que si bien el efecto del videojuego no se relaciona directamente con el incremento de las inteligencias, si puede contribuir a mejorarlas en algunos sujetos de la Fase II. Concretamente, en el GC se observa una mayor variación respecto a las desviaciones estándar que en el GE en ambas fases, lo que implica una mayor variabilidad en los valores medios de cada inteligencia en cada fase. Los resultados obtenidos en ambas fases verifican la contribución del videojuego al incremento de las IM en los alumnos de primaria al concluir la experiencia. La prueba t-student permitió comparar las medias obtenidas por la totalidad de la muestra $(\mathrm{N}=101)$ en ambas fases, observándose una mejora cuantitativa en la mayoría de las inteligencias $(p<.05)$. En conclusión, una adecuada integración curricular del videojuego puede contribuir a la mejora global de las inteligencias en los escolares de primaria.

\subsection{Diferencias entre sexos}

Un estudio más pormenorizado permite analizar la valoración media por sexo para detectar su influencia en los resultados. En la Tabla 4 se observan los datos relativos a las medidas de los sujetos del GE para cada inteligencia en ambas fases. 


\begin{tabular}{|c|c|c|c|c|c|}
\hline \multirow{2}{*}{ Inteligencia } & \multirow{2}{*}{ Género } & \multicolumn{2}{|c|}{ Fase I } & \multicolumn{2}{|c|}{ Fase II } \\
\hline & & Media & SD & Media & SD \\
\hline \multirow{2}{*}{ Lingüística } & Niños & 2.57 & 0.56 & 2.81 & 0.56 \\
\hline & Niñas & 2.64 & 0.56 & 2.91 & 0.53 \\
\hline \multirow{2}{*}{ Naturalista } & Niños & 2.56 & 0.58 & 2.93 & 0.60 \\
\hline & Niñas & 2.42 & 0.71 & 2.71 & 0.69 \\
\hline \multirow{2}{*}{ Matemática } & Niños & 2.55 & 0.52 & 2.66 & 0.57 \\
\hline & Niñas & 2.37 & 0.59 & 2.88 & 0.73 \\
\hline \multirow{2}{*}{ Visoespacial } & Niños & 2.65 & 0.44 & 2.85 & 0.52 \\
\hline & Niñas & 2.55 & 0.55 & 2.97 & 0.65 \\
\hline \multirow{2}{*}{ Musical } & Niños & 2.60 & 0.49 & 2.81 & 0.51 \\
\hline & Niñas & 2.62 & 0.55 & 2.83 & 0.55 \\
\hline \multirow{2}{*}{ Corporal } & Niños & 2.61 & 0.50 & 2.91 & 0.49 \\
\hline & Niñas & 2.45 & 0.59 & 2.71 & 0.71 \\
\hline \multirow{2}{*}{ Interpersonal } & Niños & 2.62 & 0.66 & 2.92 & 0.57 \\
\hline & Niñas & 2.57 & 0.64 & 2.80 & 0.67 \\
\hline \multirow{2}{*}{ Intrapersonal } & Niños & 2.63 & 0.47 & 2.82 & 0.42 \\
\hline & Niñas & 2.55 & 0.51 & 2.71 & 0.59 \\
\hline
\end{tabular}

Tabla 4. Medias y las desviaciones estándar de las IM del GE en ambas fases según el sexo.

En general, se detectó un incremento mayor en las niñas tras su participación en la experiencia, pues mejoran sus inteligencias más que los niños, destacándose mayores incrementos en la Matemática $(p<.001)$ y Viso-espacial $(p<.003)$. Especialmente, se observa que la Matemática mejora susceptiblemente más en ellas que en ellos.

Además, con la prueba t-student se constató que la variable curso no influye en las valoraciones registradas en las distintas inteligencias, sólo existen diferencias significativas respecto a la inteligencia Visoespacial $(p<.039)$ beneficiándose del experimento los sujetos del segundo curso.

Por otro lado, tras constatar los niveles que registran los sujetos en cada inteligencia antes y después de su participación en la experiencia con el videojuego, se analiza el comportamiento de cada uno respecto a los ítems que las explican, destacando los que mejoraron significativamente $(p<.05)$.

\section{CONCLUSIONES}

Existe un incremento general en las inteligencias de los escolares tras participar en la experiencia innovadora de integración del videojuego en el aula. Ello suscita una interesante reflexión sobre las potencialidades educativas de algunos videojuegos y sobre la oportunidad de integrarlos en las aulas, ligado a las necesidades formativas de los docentes para que aprendan 
a seleccionarlos e incluirlos en sus aulas aprovechando la motivación extrínseca que suponen los incentivos multisensoriales de algunos serious games para ponerlos al servicio de la adquisición de destrezas y habilidades.

El GC permitió evidenciar que el videojuego Naraba sí influye en la mejora de las inteligencias de los sujetos, puesto que en este grupo a excepción de la musical (que fue significativa en las niñas) las inteligencias no mostraron una mejora significativa.

La lingüística y la viso-espacial presentan los mayores incrementos estadísticamente significativos al finalizar la experiencia, refrendados con la mejora cualitativa percibida por los docentes en la lecto-escritura gracias al videojuego: lectura de subtítulos e instrucciones del mismo, realización de las actividades del taller de escritura. Algo predecible dada su naturaleza y desafíos: recuerdo de nombres de personajes, lugares de origen, cómo son, a qué se dedican, nombres de las islas y de sus criaturas fantásticas... Desarrollando su capacidad memorística, comprensión oral y escrita y adquisición de nuevo vocabulario.

Es importante destacar que el videojuego contribuye a la mejora de la inteligencia lingüística, especialmente en relación al fomento del gusto por la lectura.

Al tratarse de un videojuego en 3D que exige habilidades espacialmente para orientarse, se esperaba que la inteligencia viso-espacial resultara incrementada en mayor medida, pero sólo aumentaron los componentes referidos a la visualización de imágenes, animaciones y objetos de la pantalla.

Los componentes de la naturalista que se incrementan son los vinculados a los contenidos específicos del videojuego y a su presentación. Las misiones del videojuego relacionadas con la naturaleza son las más atractivas, implican comparación o clasificación de objetos, líquidos, especies animales, etc., competencias propias de esta inteligencia. La posibilidad de experimentar con líquidos, mezclas, transformaciones... facilita la simulación de fenómenos que normalmente no se dan en la realidad del aula.

La mayoría de los componentes que explican la inteligencia matemática se incrementaron debido al entrenamiento adquirido con los rompecabezas, cálculos y categorización del videojuego. Ello permitió utilizarlo como herramienta de aprendizaje y refuerzo de contenidos matemáticos.

Asimismo, el taller de composición musical del videojuego y las actividades relacionadas con ritmos y clasificación de sonidos, favorecieron el incremento de la inteligencia musical, al memorizar ritmos y melodías para reproducirlas después, luego, no sorprende que el componente que mayores cambios presenta sea el referido al recuerdo de melodías y canciones.

La corporal-cinestésica se desarrolló conjuntamente con la viso-espacial, al simular movimientos del avatar virtualmente con el ratón y el teclado. Sin embargo, la movilidad física de los jugadores era reducida, lo cual explica que la corporal apenas se potenciara. 
La observación directa permitió constatar el fomento de actitudes de apoyo y colaboración mutua entre los alumnos durante la experiencia con el videojuego, evidenciándose que los "más expertos" aconsejaban a los que tenían dificultades. Aspectos que explican el incremento de la inteligencia interpersonal al concluir la experiencia. También se debe señalar que el juego individual permitió desarrollar la autonomía de los alumnos incrementando su inteligencia intrapersonal. Los éxitos y fracasos ayudaron a crear un clima de esfuerzo, superación y afán por adquirir nuevos aprendizajes para avanzar en el juego.

Tras los resultados obtenidos, los serious games pueden considerarse unos formatos idóneos tanto para favorecer la inteligencia matemática y la viso-espacial en las niñas, al situar las actividades en escenarios tangibles que minimizan el grado de abstracción requerido para asimilar determinados conceptos; como para desarrollar la inteligencia lingüística y la interpersonal en los niños, al ofrecerles oportunidades para elaborar relatos a partir de las imágenes presentadas así como para compartir experiencias con otros en busca de la resolución de problemas colaborativamente.

Sin embargo, el éxito de este tipo de experiencias innovadoras apoyadas en videojuegos radica en la cualificación de los docentes para que sepan integrar adecuadamente estos recursos en el aula, solventando las diferentes problemáticas que ello genera.

Finalmente, hay que subrayar que la nueva tendencia del Aprendizaje basado en juegos reclama por un lado, de nuevas formas de alfabetización, de intercambio comunicativo y de pensamiento para explotar estos instrumentos lúdicos digitales en el aula. Y por otro, de la convergencia del sector educativo y el empresarial para diseñar videojuegos atractivos cuyo motor de juego se ponga al servicio del aprendizaje y potencien las ocho inteligencias.

Tras los resultados obtenidos, se puede concluir que los serious games pueden considerarse unos formatos idóneos tanto para favorecer la inteligencia matemática y la viso-espacial en las niñas, al situar las actividades en escenarios tangibles que minimizan el grado de abstracción requerido para asimilar determinados conceptos; como para desarrollar la inteligencia lingüística y la interpersonal en los niños, al ofrecerles oportunidades para elaborar relatos a partir de las imágenes presentadas así como para compartir experiencias con otros en busca de la resolución de problemas colaborativamente.

Sin embargo, el éxito de este tipo de experiencias innovadoras apoyadas en videojuegos radica en la cualificación de los docentes para que sepan integrar adecuadamente estos recursos en el aula, solventando las diferentes problemáticas que ello genera.

Finalmente, hay que subrayar que la nueva tendencia del Aprendizaje basado en juegos reclama por un lado, de nuevas formas de alfabetización, de intercambio comunicativo y de pensamiento para explotar estos instrumentos lúdicos digitales en el aula. Y por otro, de la convergencia del sector educativo y el empresarial para diseñar videojuegos atractivos cuyo motor de juego se ponga al servicio del aprendizaje y potencien las ocho inteligencias. 


\section{REFERENCIAS}

Amstrong, T. (1994). Multiple intelligences in the classroom. Virginia: ASCD.

Basak, C., Boot, W. R., Voss, M.W., \& Kramer, A. F. (2008). Can training in a real-time strategy video game attenuate cognitive decline in older adults? Psychology \& Aging, 23(4), 765777.

Botero, J. A. (2011). Propuesta de un juego de rol para evaluar la competencia del liderazgo basado en el método de desarrollo de habilidades gerenciales. (Tesis doctoral inédita). Universidad Nacional de Colombia. Medellín.

Castronova, E. (2006). On the Research Value of Large Games: Natural Experiments in Norrath and Camelot. Games \& Culture, 2(1), 163-186.

Chang, K. E., Wu, L. J., Weng, S. E., \& Sung, Y. T. (2012). Embedding game-based problem-solving phase into problem-posing system for mathematics learning. Computers \& Education, $58(2), 775-786$.

Cortés, S., García, M. R., \& Lacasa, P. (2012). Videojuegos y Redes Sociales. El proceso de identidad en Los Sims 3. RED. Revista de Educación a Distancia, 33. Recuperado de http://www.um.es/ead/red/33/

Del Castillo, H., Herrero, D., García, A. B., Checa, M., \& Monjelat, N. (2012). Desarrollo de competencias a través de los videojuegos deportivos: alfabetización digital e identidad. RED. Revista de Educación a Distancia, 33. Recuperado de http://www.um.es/ead/red/33/

Del Moral, M. E. (2012). Desarrollo de las inteligencias múltiples apoyado en videojuegos. Ponencia impartida en el I Congreso Internacional de Videojuegos y Educación, 2-3 febrero, 2012. Alfás del Pi (Alicante): Universidad de Valencia. Recuperado de http://www.uv.es/ordvided/ACTAS/ACTAS\%20CIVE\%202012.pdf

Del Moral, M. E., \& Villalustre, L. (2012). Videojuegos e infancia: análisis, evaluación y diseño de videojuegos desde una perspectiva educativa. En García Jiménez, A. (Ed.). Comunicación, Infancia y Juventud. Situación e Investigación en España. (pp. 97-112). Barcelona: UOC.

Echeverría, A., García-Campo, C., Nussbaum, M., Gil, F., Villalta, M., Améstica, M., \& Echeverría, S. (2011). A framework for the design and integration of collaborative classroom games. Computers \& Education, 57(1), 1127-1136.

Ferrándiz, C., Hernández, D., Berjemo, R., Ferrando, M., \& Sáinz, M. (2012). La inteligencia emocional y social en la niñez y adolescencia: validación de un instrumento para su medida en lengua castellana. Revista de Psicodidáctica, 17(2), 309-338. Recuperado de http://www.ehu.es/ojs/index.php/psicodidactica/article/view/2814/5953 
García, M. R., Cortés, S., \& Martínez, R. (2011). De los videojuegos comerciales al currículum: Las estrategias del profesorado. Revista Icono14, 9(2), 249-261. Recuperado de http://www.icono14.net/ojs/index.php/icono14/article/view/49/48

Gardner, H. (2005). Inteligencias múltiples. La teoría en la práctica. Barcelona: Paidós.

Gómez del Castillo, M. T. (2007). Videojuegos y transmisión de valores. Revista Iberoamericana de Educación, 43(6), 1-10.

Graf, D. L., Pratt, V. L., Hester, C. N., \& Short, K. R. (2009). Playing active video games increases energy expenditure in children. Pediatrics, 124(2), 534-540.

Greenfield, P. M. (2009). Technology and informal education: What is taught, what is learned. Science, 323(2), 69-71.

Greitemeyer, T., \& Osswald, S. (2010). Effects of prosocial video games on prosocial behavior. Journal of Personality \& Social Psychology, 98(2), 211-221.

Korallo, L., Foreman, N., Boyd-Davis, S., Moar, M., \& Coulson, M. (2012). Do challenge, task experience or computer familiarity influence the learning of historical chronology from virtual environments in 8-9 year old children? Computers \& Education, 58(4), 11061116.

Llorca, M. A. (2009). Hábitos y uso de los videojuegos en la comunicación visual: influencia en la inteligencia espacial y el rendimiento (Tesis inédita). Universidad de Granada. Granada.

“Ley orgánica 2/2006, de 3 de mayo, de Educación (LOE)". Boletín Oficial del Estado, 106, (4 de mayo de 2006), págs. 17158-17207.

Marín, V., \& García, M. D. (2005). Los videojuegos y su capacidad didáctico-formativa. Pixel-Bit. Revista de Medios y Educación, 26, 113-119.

Micronet (2010). Naraba World. Recuperado de http://www.narabaworld.com/

Papastergiou, M. (2009). Exploring the potential of computer and video games for health and physical education: A literature review. Computers \& Education, 53(3), 603-622.

Pindado, J. (2005). Las posibilidades educativas de los videojuegos. Una revisión de los estudios más significativos. Pixel-Bit. Revista de Medios y Educación, 26, 55-67.

Prieto, M. D., \& Ballester, P. (2003). Las inteligencias múltiples. Diferentes formas de enseñar y aprender. Madrid: Ediciones Pirámide.

Prieto, M. D., \& Ferrándiz, C. (2001). Inteligencias múltiples y currículum escolar. Málaga: Ediciones Aljibe. 
Riha, M., \& Robles-Piña, R.A. (2009). The Influence of Multiple Intelligence Theory on WebBased Learning. MERLOT. Journal of Online Learning \& Teaching, 5(1), 97-103. Recuperado de http://jolt.merlot.org/vol5no1/robles-pina 0309.pdf

Robertson, J. (2013). The influence of a game-making project on male and female learners' attitudes to computing. Computer Science Education, 23(1), 58-83.

Sanford, K., \& Madill, L. (2007). Understanding the Power of New Literacies through Video Game Play and Design. Canadian Journal of Education, 30(2), 432-455.

Saricaoglu, A, \& Arikan, A. (2009). A study of multiple intelligences, foreign language success and some selected variables. Journal of Theory \& Practice in Education, 5(2), 110-122. Recuperado de http://www.bcl.edu.ar/spip/IMG/pdf/asaricaoglu aarikan12.pdf

Schaaf, R. (2012). Does digital game. based learning improve student time-on-task behavior and engagement in comparison to alternative instructional strategies? Canadian Journal of Action Research, 13(1), 50-64. Recuperado de http://cjar.nipissingu.ca/index.php/cjar/article/view/30/27

Sung, H., \& Hwang, G. (2013). A collaborative game-based learning approach to improving students' learning performance in science courses. Computers \& Education, 63(1), 43-51.

Thorne, S. L., Black, R. W., \& Sykes, J. M. (2009). Second Language Use, Socialization, and Learning in Internet Interest Communities and Online Gaming. The Modern Language Journal, 93(1), 802-821.

Vos, N., van der Meijden, H., \& Denessen, E. (2011). Effects of constructing versus playing an educational game on student motivation and deep learning strategy use. Computers \& Education, 56(1), 127-137.

Yang, J. C., \& Chen, S. Y. (2010). Effects of gender differences and spatial abilities within a digital pentominoes game. Computers \& Education, 55(3), 1220-1233.

\section{Para citar este artículo:}

Del-Moral-Pérez, M. E., Guzmán-Duque, A. P. \& Fernández, L.C. Serious Games: escenarios lúdicos para el desarrollo de las inteligencias múltiples en escolares de primaria. EDUTEC, Revista Electrónica de Tecnología Educativa, $47 . \quad$ Recuperado el $\mathrm{dd} / \mathrm{mm} / \mathrm{aa}$ de http://edutec.rediris.es/Revelec2/Revelec47/n47 DelMoral-Guzman-Fernandez.html 